\title{
Varieties of Disagreement and Predicates of Taste
}

\author{
Torfinn Thomesen Huvenes \\ Penultimate Draft \\ Published in Australasian Journal of Philosophy, vol. 90, 2012, pp. 167-181.
}

\begin{abstract}
Predicates of taste, such as 'fun' and 'tasty', have received considerable attention in recent debates between contextualists and relativists, with considerations involving disagreement playing a central role. Considerations involving disagreement have been taken to present a problem for contextualist treatments of predicates of taste. My goal is to argue that considerations involving disagreement do not undermine contextualism. To the extent that relativism was supposed to be motivated by contextualists being unable to deal with disagreement, this motivation is lacking. The argument against contextualism rests on a too simple and narrow conception of disagreement that turns out to be problematic once we consider a wider range of cases. If we reject the assumptions about disagreement that the argument rests on, it no longer poses a threat to contextualism.
\end{abstract}

Keywords: Contextualism, relativism, disagreement, predicates of taste.

\section{Introduction}

Supporters of relativism, such as Kölbel [2002, 2004], Lasersohn [2005], MacFarlane [2007] and Stephenson [2007], have argued that contextualist treatments of predicates of taste fail to deliver the right predictions about when speakers disagree. Instead they argue that we should prefer a relativist treatment of predicates of taste. I will argue that this line of argument is not as effective as it initially appears to be. My main concern in this paper is to argue that there are other cases of disagreement that we need to take into account. The examples I have in mind are examples like (1). 
b. Mark: I disagree, it's too hot for me.

I will argue that examples like this do not call for a relativist treatment. Moreover, once examples like (1) have been taken into account, the cases of disagreement that were supposed to present a problem for contextualism seem to be less surprising from a contextualist point of view.

In section 2 I outline the difference between contextualist and relativist treatments of predicates of taste. In section 3 I explain why contextualist treatments allegedly have problems with disagreement. In section 4 I present some further disagreement data and argue that the cases that were allegedly problematic from a contextualist point of view are less surprising in light of the new data. In section 5 I try to assuage some doubts one might have about the data presented in section 4 . In section 6 I argue that focusing on other disagreement markers does not make the argument against contextualism more effective. In section 7 I offer some preliminary remarks about the possibility of understanding the disagreement data in terms of conflicting non-doxastic attitudes.

\section{Contextualism and Relativism}

The debate between contextualists and relativists that I am concerned with is a debate about the semantics of predicates of taste. In terms of characterizing these predicates, I will be content with saying that they are formed from adjectives such as 'fun' and 'tasty'. Instead of trying to come up with criteria for identifying the relevant expressions, I will follow Lasersohn and most of the other participants in this debate and focus on the expressions 'fun' and 'tasty'.

It is common ground between contextualists and relativists that there is some sort of contextual variation in the truth-values of sentences containing 'fun' and 'tasty'. The contextualist and relativist treatments of predicates of taste that I am interested in take the truth-values of such sentences to depend on whose taste that is relevant in some way or another. However, they differ with respect to how they account for this variation in truth-values. According to the semantic framework of Kaplan [1989], which I will be assuming for the purpose of this discussion, the content of an expression in a 
context can be represented as a function from circumstances of evaluation, world-time pairs on his view, to extensions. The extension of a sentence is a truth-value and the truth-value of a sentence can depend on both the context insofar as it plays a role in determining the content and on the circumstance of evaluation with respect to which we evaluate the content.

Simply put, contextualism about predicates of taste is the thesis that predicates of taste, or the sentences that contain them, have different contents in different contexts. According to the kind of contextualist position we are concerned with here, the content will vary depending on whose taste that is relevant. I understand this in a relatively broad sense in which it is compatible with, but does not require that the context-dependence is traceable to a covert element in the logical form. What matters is that the variation in truth-value is explained by a variation in content.

The relativist account differs from the contextualist account in that it does not appeal to a contextual variation in the content of sentences containing predicates of taste, but instead takes the contents of such sentences to be true or false relative to some appropriate parameter other than worlds and times. ${ }^{1}$ Following Lasersohn [2005], relativists about predicates of taste can take the truth or falsity of sentences containing predicates of taste to be relative to individuals. In terms of modifying the basic semantic framework from Kaplan this means that circumstances of evaluation are treated as world-time-individual triples rather than world-time pairs. The upshot is that there can be a variation in truth-value without a variation in content.

\section{The Problem of Disagreement}

Supporters of relativism have argued that contextualism has problems making sense of disagreement involving predicates of taste. ${ }^{2}$ For instance, take a context in which Bob and Carol are riding a roller

\footnotetext{
${ }^{1}$ I am using 'relativism' in a broader sense than MacFarlane [2005, 2009] insofar as I am not distinguishing between views according to which the relevant parameters are determined by the context of utterance and views according to which they are determined by the context of assessment. Moreover, distinguishing between the context of utterance and the context of assessment also allows us to identify a view according to which the content of a sentence depends on the context of assessment. See e.g. Cappelen [2008a, 2008b] and Weatherson [2009]. Since I am primarily interested in defending a traditional form of contextualism, these distinctions are not crucial to my overall line of argument.

I also acknowledge that it is somewhat arbitrary to start out by taking circumstances of evaluation to be worldtime pairs rather than just worlds or world-time-location triples. However, as far as I can see, nothing substantial depends on this.

${ }^{2}$ For a more detailed presentation of the argument against contextualism see e.g. Lasersohn [2005].
} 
coaster. They both recognize that while Bob is enjoying the ride, Carol is not, and they have the following conversation:
a. Bob: This is fun.
b. Carol: I disagree, this is not fun.

It is easy to construct similar examples involving 'tasty'. Imagine that Bob and Carol are at a party and that the dialogue in (3) takes place as they are having some chilli.

(3) a. Bob: This chilli is tasty.

b. Carol: I disagree, this chilli is not tasty.

In both (2) and (3) it appears correct for Carol to report that she disagrees with Bob. ${ }^{3}$ However, it is not clear that contextualism respects this judgement. The worry is that if contextualism is correct and there is a variation in the content of the relevant sentences, the content of the sentence uttered by Carol will not contradict the content of the sentence uttered by Bob. In that sense, Carol will not be denying the content of the sentence uttered by Bob. But then in what sense do they disagree?

The problem becomes particularly clear if we consider a rather simple-minded version of contextualism according to which it is always just the tastes of the speaker that are relevant. ${ }^{4}$ In order to appreciate the point it is worth looking at an example which does not involve predicates of taste, but which involves the first-person pronoun 'I'. The following example is a slightly modified version of an example that Lasersohn [2005] uses to make a similar point.
(4)
a. A: I am a doctor.

\footnotetext{
${ }^{3}$ The use of 'I disagree' may be a bit stilted, but insofar as we want to focus on issues concerning disagreement it makes sense to start out by looking at cases in which one of the parties explicitly reports that she disagrees with the other party. The possible stiltedness of 'I disagree' will be relevant in section 4 and 5 when I discuss examples like (1). I will look at other disagreement markers in section 6.

${ }^{4}$ I am not going to discuss the extent to which contextualists can avoid this problem altogether by adopting a more sophisticated version of contextualism. For attempts to defend contextualism in this way see e.g. Glanzberg [2007], Recanati [2007: ch. 11], Cappelen and Hawthorne [2009] and Schaffer [forthcoming]. For critical discussion see e.g. Lasersohn [2005] and MacFarlane [2007].
} 
b. B: I disagree, I am not a doctor.

The response in (4b) is clearly inappropriate. It is hard to make sense of the response except as a misunderstanding of what the speaker of (4a) said. If the simple-minded version of contextualism was correct, we might expect (2b) and (3b) to be bad in the same way as (4b), but that does not seem to be the case.

On the other hand, if relativism is correct, the content of $(2 a)$ and $(2 b)$ cannot both be true relative to the same circumstance of evaluation and in that sense the content of (2a) contradicts the content of (2b). While more needs to be said before it becomes clear that relativism offers a genuine explanation of the apparent disagreement in (2) and (3), the main question that I am interested in is how problematic examples like (2) and (3) are from a contextualist point of view. ${ }^{5}$ I will argue that these examples are less problematic from a contextualist point of view once we take into account a sufficiently broad range of cases of disagreement.

\section{More Disagreement Data}

As it has been presented here, the argument against contextualism rests on the assumption that Carol must be denying the content of the sentence uttered by Bob in order for them disagree. While I am going to argue that we have good reasons to reject this assumption, it might seem plausible if one is only looking at a fairly limited range of cases of disagreement. It is perhaps tempting to think that the disagreement in examples like (2) and (3) should be understood in more or less the same way as the disagreement in examples like (5).

a. Robert: The Cuban missile crisis took place in 1963.

b. Bobby: I disagree, it took place in 1962, not 1963.

On the assumption that Robert and Bobby are talking about the actual history of the world and are referring to the same historical event, I think it is safe to say that Robert and Bobby disagree about

\footnotetext{
${ }^{5}$ For a discussion of how to develop a relativist account of disagreement see e.g. MacFarlane [2007].
} 
when the Cuban missile crisis took place. By uttering (5b), Bobby is denying the content of (5a), the sentence uttered by Robert. More precisely, the content of the sentence uttered by Bobby and the content of the sentence uttered by Robert cannot both be true.

This is a clear and uncontroversial example of disagreement. Indeed, it might be tempting to think that we can understand all cases of disagreement in dialogues of this form along the same lines as (5), that is, in terms of one of the speakers denying the content of the sentence uttered by the other speaker. However, it does not seem that we can fit all cases of disagreement into this mould. I am primarily interested in examples like (1).
a. Sally: I like this chilli.
b. Mark: I disagree, it's too hot for me.

We can imagine the conversation in (1) as taking place while Sally and Mark, like Bob and Carol, are having some chilli at a party. I take it that Mark disagrees with Sally when he asserts (1b) in response to (1a) even though the truth of (1a) as uttered by Sally is consistent with the truth of (1b) as uttered by Mark. If Mark was denying the content (1a) he would have to think that Sally was somehow insincere or mistaken or confused about her own tastes. Apart from this being odd under normal circumstances, it would be hard to make sense of him saying that the chilli is too hot for him. In other words, we seem to have a case of disagreement in which Mark is not denying the content of the sentence uttered by Sally.

Judgements about examples like (1) are liable to vary to some extent. Even though some find Mark's use of 'I disagree' a bit stilted, I will assume for now that we want to take the example at face value and say that Sally and Mark disagree. ${ }^{6}$ In section 5 I will address some potential concerns about examples like (1) and argue that such examples should be taken seriously.

Examples like (1), if taken at face value, are relevant in the context of evaluating the extent to which examples like (2) and (3) are problematic from a contextualist point of view. Insofar as it is

\footnotetext{
${ }^{6}$ Even if we grant that there may be something stilted about the use of 'I disagree' in (1), it is not clear what this shows. As noted in footnote 3, the point that the use of 'I disagree' may be stilted does not only apply to examples like (1). These are among the issues that will be addressed in section 5 .
} 
being assumed that the cases of disagreement that are allegedly problematic from a contextualist point of view have to be understood on the model of (5), examples like (1) show that this cannot be true in general. It is not always the case that disagreement in dialogues of this form is a matter of one of the speakers denying the content of the sentence uttered by the other speaker. ${ }^{7}$

Furthermore, examples like (2) and (3) are much less surprising from a contextualist point of view if we take examples like (1) at face value. There are two important points here. The first point to note is that examples like (1) do not lend any kind of support to relativism. Indeed, it is not clear how a plausible account of this kind of disagreement along relativist lines could be constructed. While there may be difficult questions pertaining to the correct semantics for 'likes', there does not seem to be any further need for a relativist or contextualist semantics of the sort that has been proposed for predicates of taste. This means that we have to look elsewhere for an explanation of the disagreement in examples like (1).

The second point is that if we find disagreement when speakers use sentences like 'I like this chilli' to express their personal tastes, it is not surprising, even from a contextualist perspective, that the same is true in the cases involving 'fun' and 'tasty'. If Mark and Sally can disagree even though Mark is not denying the content of the sentence uttered by Sally, it is not surprising that the same is true in the case of Bob and Carol. It seems reasonable to assume that whatever the right explanation of Sally and Mark's disagreement in (1) is, that explanation can be extended to cover examples like (2) and (3) as well.

If all of this is correct, then it seems to undermine the argument against contextualism. The presence of disagreement in cases involving 'fun' and 'tasty' is to be expected given that we have disagreement in cases involving 'likes'. Moreover, the presence of disagreement in the latter case does

\footnotetext{
${ }^{7}$ Examples like (1) are not the only examples of disagreement that cannot easily be understood along the same lines as (5). Suppose that Pierre and Marie are conducting a series of experiments in order to test a scientific hypothesis that they both believe to be false and that the following conversation takes place after one of the experiments:

Pierre: The hypothesis is false.

Marie: I disagree, we need to do further testing.

In this case we do not need to understand Marie as denying the content of the sentence uttered by Pierre. Indeed, since she herself believes that the hypothesis is false, and thus accepts the content of the sentence uttered by Pierre, it is more plausible to take her to disagree with Pierre on the grounds that there is insufficient evidence for him to assert that the hypothesis is false without further testing. In other words, Pierre and Marie disagree even though Marie is not denying the content of the sentence uttered by Pierre.
} 
not call for a relativist explanation and once this is recognized it is hard to see why it should do so in the former case either. At least the relativists owe us an account of why the cases should be treated differently.

This does not mean that there is nothing to be gained by pursuing other ways of defending contextualism. In particular, it might still be a good idea to improve on the simple-minded version of contextualism according to which it is only the tastes of the speaker which matters. What is relevant may be the tastes of the members of some group that is determined by the context and not just the tastes of the speaker. For instance, it may sometimes be the standards of the wider community that are relevant [Recanati 2007: ch. 11]. This would make it easier to explain why we take Bob and Carol to disagree, because we can interpret them as making claims about what is fun and tasty for members of their community. Still, I think it is important to recognize that contextualists are not necessarily committed to thinking about the disagreement in these terms. Bob and Carol may disagree even if Carol is not denying the content of the sentence uttered by Bob. This gives contextualists a certain amount of flexibility when it comes to responding to worries about disagreement.

\section{Doubts about the Data}

All of this requires that one takes examples like (1) at face value. While I am not alone in drawing attention to examples of disagreement like $(1)^{8}$, some may be inclined to dismiss examples like (1) outright and argue that, contrary to appearances, Sally and Mark do not disagree after all. In response to an objection along these lines it makes sense to compare examples like (1) with examples in which it really does seem wrong to report that the parties disagree. While judgements about the appropriateness of Mark's response in (1) might be somewhat delicate, it is useful to consider the difference between (1) and (4).
(4)
a. A: I am a doctor.
b. B: I disagree, I am not a doctor.

\footnotetext{
${ }^{8}$ See e.g. Weatherson [2009] who points out that this sort of disagreement is known from debates about noncognitivism in moral philosophy.
} 
As noted earlier, the response in (4b) is clearly inappropriate and it is hard to make sense of it except as a misunderstanding. On the other hand, dialogues like (1) do not seem to exhibit this kind of inappropriateness. We do not have the same problem making sense of Mark's response to Sally in (1). I take this to count against someone who wants to say that there is no disagreement in examples like (1). At the very least, one would have to explain why Mark's response in (1) is more natural and appropriate than the response in (4).

In section 4 I acknowledged that some find the use of 'I disagree' in (1) a bit stilted. But to the extent that I am willing to grant that, I also think that to some extent can be said of (2) and (3) as well. ${ }^{9}$ It is not clear that there are sufficient grounds for dismissing examples like (1) while continuing to treat examples like (2) and (3) as evidence against contextualism. In general it is problematic to be dismissive of examples like (1) if one wants to make positive use of other disagreement data. There might very well be reasons to conclude that examples like (1) are not cases of genuine or real disagreement in some interesting sense, but then we should be prepared to say the same thing about other putative cases of disagreement as well. In this context it is also worth noting that some philosophers have expressed doubts about the judgements about disagreement that relativists rely on [Cappelen and Hawthorne 2009: ch. 4; Stanley 2005: 144]. While I am not advocating this line of response on behalf of contextualism, it makes it somewhat awkward from a dialectical point of view for relativists to be dismissive of other putative cases of disagreement. In the absence of a more significant difference between examples like (1) and examples like (2) and (3), it is not clear that relativists are warranted in dismissing examples like (1) while treating examples like (2) and (3) as unproblematic.

Even if one does not want to outright dismiss examples like (1), one might think that since the denial in (1b) cannot plausibly be interpreted as targeting the content of (1a) it could target some other content conveyed by Sally's utterance. Perhaps a relativist could argue that Sally's utterance conveys the content that the chilli is tasty, with this being understood in relativist terms as having different

\footnotetext{
${ }^{9}$ The point that the use of 'I disagree' may also be a bit stilted in the case of examples like (2) and (3) was made in footnote 3 .
} 
truth-values relative to different individuals. Insofar as relativists are in a position to explain the disagreement in examples like (2) and (3), they could extend that explanation to examples like (1). On this picture relativism would be needed to make sense of the disagreement after all.

While this is an interesting suggestion that deserves further investigation, there are some questions that need to be addressed. For one thing it is not obvious why we should take Sally to convey this particular content by her utterance. It is tempting to think of this in terms of a conversational implicature, but how does this implicature arise? ${ }^{10}$ A natural suggestion is to appeal to considerations involving relevance. While there might be other explanations available, this looks like the most obvious strategy. ${ }^{11}$ The basic idea is that what is relevant is whether the chilli is tasty and not just whether Sally likes it and that is why she is understood as conveying that the chilli is tasty.

However, given the context in which Sally utters (1a), it is not clear why her contribution to the conversation should not count as sufficiently relevant unless she is understood as conveying something beyond that she likes the chilli, and more specifically, that the chilli is tasty. If they had been talking about what kind of food they find tasty, that might be a reasonable assumption, but that does not have to be the case. They could just as well have been talking about what they like about the party or what is happening there. Moreover, this does not seem to affect the judgement that Sally and Mark disagree in (1). This is not to rule out an explanation in terms of relevance, but only to say that the availability of such an explanation cannot be taken for granted.

Another question is whether the presence of a conversational implicature would be sufficient to explain the disagreement data. It is not clear whether it is always appropriate to use 'I disagree'

\footnotetext{
${ }^{10}$ An anonymous referee pointed out to me that the dialogue in (1) gets worse if we replace 'it's too hot for me' with 'I am tired' even though the latter may also be a good reason for not enjoying the chilli. A possible explanation is that it is the taste of the chilli and being tired does not bear on that. However, even if it is true that the taste of the chilli is somehow at stake, it is far from clear that this shows that that an account of the sort that would favour the relativists is correct.

The same referee suggested that another possible relativist response is to say that (1a) entails that Sally finds the chilli tasty and that allows the denial to target the content that the chilli is tasty. I will continue to focus on implicature-based proposals, but it is worth noting that this is might be an interesting alternative. However, more work remains to be done before it is clear that the suggestion is plausible and I will not pursue it here.

${ }^{11}$ If we take Grice's [1989] original framework as a starting point, it seems natural to appeal to the maxim of Relation. As far as the maxim of Quality and the maxim of Manner are concerned, Sally's contribution to the conversation is presumably both true and based on good evidence, and it is not obscure or ambiguous. Matters are more complicated in the case of the maxim of Quantity. However, to the extent that it makes more sense to appeal to the maxim of Quantity, this strategy seems to face the same questions as a strategy based on the maxim of Relation, In particular, it is not clear why Sally's contribution to the conversation should not count as sufficiently informative even if she is only conveying that she likes the chilli.
} 
when rejecting an implicature. Let us consider a case inspired by one of Grice's [1989: 37] original examples. A and B are private detectives hired by Mrs. X to spy on her husband, Mr. X, and they are talking about what he is up to.
a. A: Mr. X is meeting a woman tonight.
b. B: I disagree, he is meeting his wife.

Following Grice, I take it that $\mathrm{A}$ is implicating that the woman $\mathrm{Mr}$. $\mathrm{X}$ is meeting is not his wife, and $\mathrm{B}$ is naturally understood as rejecting that implicature. Still, there seems to be something awkward about B's use of 'I disagree' in (6b). These judgements are admittedly fairly subtle and I am reluctant to put too much weight on a single example, but the point is to raise a question about whether the use of 'I disagree' is always appropriate when rejecting an implicature in this way. If it is not, there is at least some additional work to be done to explain why it is appropriate in the case of (1). If one is not impressed by examples like (1) this may not be a particularly pressing worry, but insofar as one finds Mark's response in (1) appropriate and the response in (6) inappropriate, this is something that deserves further investigation. ${ }^{12}$

These considerations are not meant to rule out the possibility of saying that what is going on in (1) is that Mark is rejecting an implicature. The point is that relativists are not entitled to assume that the disagreement can be explained in this way. There are questions that need to be answered and it remains to be seen whether there is a satisfactory story to be told.

\section{Other Disagreement Markers}

Even if one accepts a lot of what I have said so far, there is still a worry that needs to be addressed. The worry is that I have been focusing on the wrong sort of data and that the relevant data involve a more restricted notion of disagreement. The cases which I have been concerned with involve the use of

\footnotetext{
${ }^{12}$ This does not amount to the claim that the use of 'I disagree' is never appropriate when targeting an implicature. Given that there are significant differences between different kinds of implicatures, it would perhaps not be surprising to find that the use of 'I disagree' is more or less appropriate in cases involving other kinds of implicatures. However, that would only illustrate the need to get clear on the details of the account.
} 
'I disagree', but there could be other disagreement markers in English that are more suited to the argumentative purposes of the relativists by allowing us to focus on a more restricted notion of disagreement. While relativists do not have to claim that the relevant notion of disagreement is always tied to the use of a specific disagreement marker, it would be useful for them if they had a way of showing that our judgements are tracking the relevant notion of disagreement. If it turns out that our judgements about cases involving other disagreement markers differ from our judgements about cases involving 'I disagree', there might be a concern that it will be difficult to extend the contextualist response that I have proposed to these cases. I will look at two such proposals and I will argue that neither of them provides relativists with the desired results.

One option is to argue that the relevant cases involve the use of 'that's not true' or 'that's false'. Insofar as we want to focus on cases of disagreement in which what is being denied is the content of the sentence that is uttered, this looks like a plausible candidate. Furthermore, this appears promising from a relativist perspective given that Mark's response in $\left(1^{*}\right)$ is clearly inappropriate.
$\left(1^{*}\right) \quad$ a. Sally: I like this chilli.
b. Mark: That's not true, it's too hot for me.

This does not seem to get the relativist very far though. The problem with this approach is that there is also something unnatural about $\left(2^{*}\right)$ and $\left(3^{*}\right)$.
(2*) a. Bob: This is fun.
b. Carol: That's not true, this is not fun.
(3*) a. Bob: This chilli is tasty.
b. Carol: That's not true, this chilli is not tasty.

For instance, we can consider the dialogue in $\left(2^{*}\right)$ as taking place in the same context as before. Bob is enjoying the ride, whereas Carol is scared and sick and clearly not enjoying it. In this context, there 
seems to be something unnatural about Carol's response in $\left(2^{*}\right)$. Similarly, if Bob and Carol are just trying the chilli at a party, there is something awkward about Carol's response in (3*).

In this respect $\left(2^{*}\right)$ and $\left(3^{*}\right)$ differ from (2) and (3), the original examples. But $\left(2^{*}\right)$ and $\left(3^{*}\right)$ are examples of the sort that were meant to present a problem for contextualism. If these dialogues do not sound natural, I assume that this should not be taken as evidence against contextualism as this is just what we would expect if contextualism was true. Judgements may vary somewhat with respect to these examples and the extent to which the responses in $\left(2^{*}\right)$ and $\left(3^{*}\right)$ are inappropriate, but I do not take these examples to be very impressive as evidence for relativism and that is what is important for our present purposes.

While it needs to be acknowledged that $\left(1^{*}\right)$ sounds worse than $\left(2^{*}\right)$ and $\left(3^{*}\right)$, that is not necessarily unexpected from the point of view of a reasonably flexible and sophisticated version of contextualism. For instance, in the case of $\left(2^{*}\right)$ we can try to make sense of Carol's behaviour by taking her to be interpreting Bob as making a stronger claim about what is fun for both of them even if that is not natural given the context. However, in the case of $\left(1^{*}\right)$ Sally makes it explicit that she is talking about what she likes and that means that such a stronger reading is unavailable.

There are cases involving predicates of taste in which a response of this sort is more natural. ${ }^{13}$ If Bob and Carol are opening a restaurant in a reasonably civilized location and they are talking about what they are going to serve in the restaurant, it seems more natural for Carol to respond to Bob's utterance of (7a) with (7b).
a. Bob: Rotten shark is tasty.
b. Carol: That's not true, rotten shark is not tasty.

While Carol's response in (7) sounds more natural than the response in $\left(3^{*}\right)$, this is not a problem for contextualism. Given a very reasonable contextualist story this is just what we should expect. In this context, it is reasonable to think that is not just the tastes of the speaker which are relevant, but the tastes of the members of a larger community of which Carol is also a member. In this case the

\footnotetext{
${ }^{13}$ Thanks to Jonathan Schaffer for making me appreciate this point.
} 
contextualists can simply understand Carol as denying the content of the sentence uttered by Bob. Moreover, it is not entirely clear what a relativist explanation of the difference between this example and examples like $\left(2^{*}\right)$ and $\left(3^{*}\right)$ would look like. Given the relativist position we have been considering so far, there does not seem to be too much of a difference between them. In other words, there is no threat to contextualism from examples like (7), and the examples which are taken to pose a threat to contextualism, examples like $\left(2^{*}\right)$ and $\left(3^{*}\right)$, do not sound very convincing in the first place. If anything, our judgements about these cases seem to fit well with what we would expect if contextualism was true.

Having said that, there are other disagreement markers that are worth examining. Stephenson [2007] is for instance careful to point out that she is focusing on a more restricted notion of disagreement that is tied to the use of expressions like 'no' and 'nuh-uh', and she allows for the possibility that whether two speakers disagree or not is a broader phenomenon. Kölbel [2002, 2004] and Lasersohn [2005] also focus on examples of this sort. Initially this seems more promising, since there is no problem about either $(2 * *)$ or $\left(3^{* *}\right)$.

$(2 * *)$ a. Bob: This is fun.

b. Carol: Nuh-uh/No, this is not fun.

$\left(3^{* *}\right) \quad$ a. Bob: This chilli is tasty.

b. Carol: Nuh-uh/No, this chilli is not tasty.

However, it is not clear that focusing on disagreement markers like 'nuh-uh' or 'no' is going to be enough for the relativists. Consider the dialogue in $(1 * *)$.

$\left(1^{* *}\right) \quad$ a. Sally: I like this chilli.

b. Mark: Nuh-uh/No, it's too hot for me.

Again we can again imagine that Sally the conversation in $\left(1^{* *}\right)$ takes place while Sally and Mark are having some chilli at a party. Is Mark's response in (1**) acceptable? While some may be uneasy 
about this example, the response does not seem to be altogether unnatural in the relevant context. Moreover, as I stressed earlier, I think it is important to compare the relevant examples with examples $\operatorname{such}$ as $\left(4^{* *}\right)$.

$\left(4^{* *}\right) \quad$ a. A: I am a doctor.

b. B: Nuh-uh/No, I am not a doctor.

I take the dialogue in $\left(1^{* *}\right)$ to be more natural than the one in $\left(4^{* *}\right)$, even though one might expect $\left(1^{* *}\right)$ to be just as bad as $\left(4^{* *}\right) .{ }^{14}$ This is at least something which requires explanation and there does not seem to be any reason to think that this explanation would ultimately give us any reason for preferring relativism over contextualism, for reasons that we have already discussed.

In the end, it I am far from convinced that the use of 'no' or 'nuh-uh' as a disagreement marker instead of 'I disagree' makes enough of a difference for the relativist to be able to mount an effective argument against contextualism. There are bound to be issues regarding when it is preferable to use 'I disagree' and when it is preferable to use 'no' and 'nuh-uh', but in the absence of more clearcut data I would be wary of investing too much in this distinction. On the overall assessment of the disagreement data considered in this section, I do not think that contextualism fares too badly. When it is less natural to use a disagreement marker in examples like (1), as in the case of 'that's not true', it also less natural in examples like (2) and (3). However, when it is more natural to use a disagreement marker in examples like (2) and (3), as in the case of 'no', that is also true of examples like (1). While I do not pretend that these matters are in any way clear-cut, I do not see a good case for relativism here.

\section{Conflicting Attitudes}

\footnotetext{
${ }^{14}$ An anonymous referee reported finding $\left(1^{* *}\right)$ just as bad as $\left(4^{* *}\right)$. I do not agree with this judgement, but I do not have a lot to say about it. I acknowledge that these judgements are not as straightforward as one would wish. Sometimes we might be in the unfortunate situation of being left with conflicting judgements about particular examples.
} 
My main concern in this paper is to argue that once we take examples like (1) into account, examples like (2) and (3) are less problematic from a contextualist point of view. While this does not presuppose any specific explanation of the data, I want to offer some speculations as to what an explanation that is compatible with a contextualist treatment of predicates of taste might look like. The idea I am interested in is to view disagreement as a matter of the parties having incompatible or conflicting attitudes. Two parties disagree just in case there is something that they have conflicting attitudes towards. This sometimes means that there is a content that one party accepts and the other party rejects, but that does not always have to be the case. Just as two parties may have conflicting beliefs, they may also have conflicting desires or preferences.

A detailed examination of the relevant issues is beyond the scope of this paper and the goal is only to present the basic idea. Still, if it these speculations turn out to be on the right track, it would lend further support to the main point by showing that there is a way of thinking about the disagreement in the relevant examples that does not favour relativism, or any other semantic theory for that matter.

To the extent that disagreement may involve different sorts of attitudes there is a sense in which there are varieties of disagreement. It would therefore be a mistake to think that all disagreement should be understood in exactly the same way as examples like (5) in which there is a content that one party accepts and the other party rejects. Furthermore, having a more liberal view of disagreement makes it easier to make sense of puzzling examples like (1). If this suggestion is on the right track, we can say that Sally and Mark disagree in virtue of having conflicting attitudes towards the chilli, and not in virtue of Mark denying the content of the sentence uttered by Sally. We can also approach Bob and Carol's disagreement in (2) and (3) in much the same way since they presumably have conflicting attitudes towards the roller coaster ride and the chilli. On this picture it is the attitudes of the parties that matter and this way of thinking of the disagreement is to a large extent independent of the semantic issues. In particular, it does not matter whether we adopt a contextualist or relativist semantics for predicates of taste.

There is something prima facie plausible about the thought that the disagreement in (1) has something to do with Sally liking the chilli and Mark disliking the chilli. Moreover, this way of 
thinking about disagreement is not entirely without precedent. It is similar to ideas usually associated with expressivist theories of the sort that figure prominently in debates in moral philosophy. ${ }^{15}$ Roughly speaking, expressivists take certain expressions to express attitudes, say, approval or disapproval, praise or resentment. This can be contrasted with the sort of truth-conditional semantics that I have been presupposing throughout this discussion, according to which the content of an expression is represented by a function from circumstances of evaluation to extensions. ${ }^{16}$ Since I am mainly interested in the debate between contextualists and relativists, I do not want to enter into a discussion about whether expressivism could provide a viable alternative to a contextualist or relativist semantics for predicates of taste. However, it is interesting to note that expressivists have claimed to be in a good position to make sense of disagreement by taking it to involve a conflict of attitudes in much the same way that I am suggesting. ${ }^{17}$

Having said that, it is important to recognize that thinking about disagreement in this way still does not force us to adopt a particular semantic theory. One can think about disagreement in this way without endorsing expressivism. In order for the speakers to disagree, they need to have certain attitudes, but there is no requirement that the relevant expressions express these attitudes in the sense that expressivists are interested in. ${ }^{18}$ In the case of (1), Sally is reporting that she likes the chilli and it is also reasonable to suppose that a sincere utterance of (2a) or (2b) is typically, though not invariably, accompanied by the speaker having a certain attitude towards the roller coaster ride or the chilli.

Even if this suggestion enjoys some prima facie plausibility and has some precedent in the literature on expressivism, there are many outstanding questions. For one thing, the key notion of

\footnotetext{
${ }^{15}$ See e.g. Stevenson $[1937,1944,1963]$ who distinguishes between what he calls 'disagreement in belief' and what he calls 'disagreement in attitude'. The way that I think about examples like (1) is very closely related to his notion of disagreement in attitude. See e.g. also Blackburn [1984, 1998].

${ }^{16}$ Blackburn and Gibbard are often mentioned as prominent contemporary defenders of expressivism in moral philosophy. See e.g. Blackburn [1984, 1998] and Gibbard [1990, 2003]. However, I will not delve into the subtle issues about how to interpret their views.

${ }^{17}$ Commenting on a case of moral disagreement over contraception, Blackburn claims that the expressivist theory 'locates the disagreement where it should be, in the clash of attitudes towards contraception' [1984: 168]. See also e.g. Blackburn [1998: 69].

${ }^{18}$ To the extent that expressivists are in a position to tell a plausible story about disagreement in terms of conflict attitudes, it is not clear why a contextualist, or a relativist for that matter, cannot tell more or less the same story. Dreier [1999] makes a similar point with respect to contextualism and expressivism in moral philosophy. He argues that a contextualist about moral judgements can follow the expressivist when it comes to explaining moral disagreement, for instance by talking about disagreement in attitudes or norms. See e.g. also Jackson and Pettit [1998].
} 
'conflicting attitudes' is admittedly fairly broad. I think it is fair to say that we have at least some grip on what it is for two people to have conflicting attitudes. If there is something that one party likes and the other party dislikes, there should be a reasonably clear sense in which they have conflicting attitudes. Still, it would be helpful if this notion could be clarified further. Without additional clarification it is not clear how such a broad notion could form a useful basis for an account of the relevant examples. ${ }^{19}$

\section{Conclusion}

In this paper I have sought to answer a specific challenge to contextualist treatments of predicates of taste, namely that such treatments leave us unable to account for certain cases of disagreement. It has been argued that the disagreement data favour a relativist account of predicates of taste. Contrary to this line of argument, I have attempted to show that considerations involving disagreement ultimately do not provide evidence for relativism. Once we look at a broader range of cases, the original cases of disagreement that were meant to lend support to relativism seem much less surprising from a contextualist perspective. While this does not show that a relativist treatment of predicates of taste is incorrect, I take it to be significant in light of the emphasis that relativists have placed on considerations involving disagreement.

No doubt there is much more that can be said about these matters, but I offer these considerations as a challenge to those who think that a relativist treatment of predicates of taste is to be preferred over a contextualist treatment for reasons having to do with disagreement. I also hope that what I have said suggests further avenues that can be explored in this debate, especially regarding the nature of disagreement and the possibility of developing an account of disagreement that allows disagreement to sometimes be a matter of conflicting non-doxastic attitudes. ${ }^{20}$

\footnotetext{
${ }^{19}$ Thanks to an anonymous referee who suggested an example which illustrates some of the difficult issues having to do with when it is legitimate to appeal to a conflict of attitudes when characterizing a case of disagreement. Suppose that it is possible to like or dislike something without being aware of it and that there is a machine that can detect these attitudes. If the dialogue in (1) took place solely on the basis of Sally and Mark checking the machine, it seems that Mark's response would be less natural. If that is right, there must be a difference between the cases that gives rise to different judgements about disagreement.

${ }^{20}$ Earlier versions of this paper were presented at the CSMN Colloquium (Oslo, 20th February 2008), the ArchéLogos Workshop on Varieties of Context Sensitivity (Barcelona, 14th March 2008), the Arché Contextualism and Relativism Seminar (St Andrews, 2nd April 2008), the CSMN Language and Rationality Seminar (Oslo, 4th
} 
Arché Philosophical Research Centre, University of St Andrews

Centre for the Study of Mind in Nature, University of Oslo

\section{References}

Blackburn, Simon 1984. Spreading the Word, Oxford: Clarendon Press.

Blackburn, Simon 1998. Ruling Passions, Oxford: Clarendon Press.

Cappelen, Herman 2008a. Content Relativism and Semantic Blindness, in Relative Truth, ed. Manuel García-Carpintero and Max Kölbel, Oxford: Oxford University Press: 265-86.

Cappelen, Herman 2008b. The Creative Interpreter: Content Relativism and Assertion, Philosophical Perspectives 22/1: 23-46.

Cappelen, Herman and John Hawthorne 2009. Relativism and Monadic Truth, Oxford: Oxford University Press.

Dreier, James 1999. Transforming Expressivism, Noûs 33/4: 558-72.

Gibbard, Allan 1990. Wise Choices, Apt Feelings, Oxford: Clarendon Press.

Gibbard, Allan 2003. Thinking How to Live, Cambridge, MA: Harvard University Press.

Glanzberg, Michael 2007. Context, Content, and Relativism, Philosophical Studies 136/1: 1-29.

Grice, Paul 1989. Logic and Conversation, in Studies in the Way of Words, ed. Paul Grice, Cambridge, MA: Harvard University Press: 22-40.

Jackson, Frank and Philip Pettit 1998. A Problem for Expressivism, Analysis 58/4: 239-51.

Kaplan, David 1989. Demonstratives, in Themes from Kaplan, ed. Joseph Almog, John Perry and Howard Wettstein, Oxford: Oxford University Press: 481-563.

Kölbel, Max 2002. Truth Without Objectivity, London: Routledge.

Kölbel, Max 2004. Faultless Disagreement, Proceedings of the Aristotelian Society 104/1: 53-73.

September 2008), and the XI TIF (Valencia, 12th January 2009). I am grateful to commentators and audiences on these occasions. Thanks to John Hawthorne, Max Kölbel, Francois Recanati, Anders Schoubye, Andreas Stokke, and two anonymous referees for helpful comments. I am particularly grateful to Herman Cappelen and Jonathan Schaffer for their extensive comments on earlier drafts of this paper. 
Lasersohn, Peter 2005. Context Dependence, Disagreement, and Predicates of Personal Taste, Linguistics and Philosophy 28/6: 643-86.

MacFarlane, John 2005. Making Sense of Relative Truth, Proceedings of the Aristotelian Society 105/1: 305-23.

MacFarlane, John 2007. Relativism and Disagreement, Philosophical Studies 132/1: 17-31.

MacFarlane, John 2009. Nonindexical Contextualism, Synthese 166/2, 231-50.

Recanati, Francois 2007. Perspectival Thought, Oxford: Oxford University Press.

Stephenson, Tamina 2007. Judge Dependence, Epistemic Modals, and Predicates of Personal Taste, Linguistics and Philosophy 30/4: 487-525.

Schaffer, Jonathan forthcoming. Perspective in Taste Predicates and Epistemic Modals, in Epistemic Modality, ed. Andy Egan and Brian Weatherson, Oxford: Oxford University Press.

Stanley, Jason 2005. Knowledge and Practical Interests, Oxford: Clarendon Press.

Stevenson, Charles L. 1937. The Emotive Meaning of Ethical Terms, Mind 46/181: 14-31.

Stevenson, Charles L. 1944. Ethics and Language, New Haven: Yale University Press.

Stevenson, Charles L. 1963. The Nature of Ethical Disagreement, in Facts and Values, ed. Charles L. Stevenson, New Haven: Yale University Press: 1-9.

Weatherson, Brian 2009. Conditionals and Indexical Relativism, Synthese 166/2: 333-57. 